use of immunosuppressive treatment in these patients. Nihtyanova et al. have now shown that the antiproliferative immunosuppressive agent mycophenolate mofetil could benefit patients with scleroderma.

Their retrospective analysis included data from 172 patients with diffuse cutaneous scleroderma from a single center; 109 patients received mycophenolate mofetil and 63 patients received other immunosuppressive agents. Data were collected from the start of treatment for a 5-year period, or until the last assessment date. Baseline differences between the groups included a higher proportion of renal scleroderma in mycophenolate-mofetil-treated patients than controls ( $23 \%$ versus $<5 \%$ ), and a higher proportion of pulmonary fibrosis in controls than in mycophenolate-mofetil-treated patients (14.3\% versus $7.3 \%)$.

Adverse events occurred in $12 \%$ of patients treated with mycophenolate mofetil; the most common type was gastrointestinaltract disturbances. Pulmonary fibrosis was evident in $12 \%$ and $19 \%$ of patients in the mycophenolate-mofetil-treated and control groups, respectively, at the end of the study. No significant differences in the Rodnan skin score and forced vital capacity were observed between the groups. The 5-year survival from disease onset was $95.4 \%$ and $85.7 \%$ for mycophenolate mofetil-treated and control patients, respectively, and the 5-year survival from the initiation of treatment was $91.7 \%$ and $77.8 \%$ in the mycophenolate-mofetil-treated and control groups, respectively.

This study had some limitations, including its retrospective design. Prospective studies to compare mycophenolate mofetil with other immunosuppressive agents should be performed.

Original article Nihtyanova SI et al. (2007) Mycophenolate mofetil in diffuse cutaneous systemic sclerosis-a retrospective analysis. Rheumatology (Oxford) 46: 442-445

\section{Lipoxins attenuate nociception at both peripheral and spinal sites}

The persistent, debilitating pain (hyperalgesia) experienced by individuals with chronic pain secondary to injury and inflammation is associated with sensitization of peripheral nociceptive terminals as well as facilitation of spinal pain signaling. Many of the factors released after tissue injury promote inflammation; however, injury also promotes the release of antiinflammatory, lipid mediators such as lipoxins, which drive resolution of inflammation. In this study, Svensson et al. investigated whether lipoxins inhibit local pain processing at the site of inflammation and at spinal sites.

Intravenous administration of lipoxins to rats before induction of hyperalgesia by intraplantar injection of carrageenan reduced subsequent thermal hyperalgesia (assessed by response latency to thermal stimulus), which demonstrated that lipoxins attenuate nociception at the inflammation site in vivo. Intrathecal (spinal) administration of lipoxin $\mathrm{A}_{4}$ to rats also attenuated carrageenan-evoked hyperalgesia and, furthermore, this antihyperalgesic effect was mediated by a mechanism distinct from that of peripheral inflammation regulation. These results suggested a spinal site of action of lipoxin $A_{4}$. Svensson et al. found that the lipoxin $\mathrm{A}_{4}$ receptor was expressed in astrocytes in rat spinal cord both in vivo and in vitro. Lastly, the authors showed that lipoxins attenuated the activation of two proinflammatory factors in astrocytesextracellular signal-regulated kinase and c-jun $\mathrm{N}$-terminal kinase, which suggested a possible mechanism for regulation of spinal nociceptive processing by lipoxins.

The presence of lipoxin $\mathrm{A}_{4}$ receptor expression in astrocytes strengthens evidence that suggests an important role for spinal nonneuronal cells in spinal facilitation of pain processing.

Original article Svensson CI et al. (2007) Lipoxins and aspirin-triggered lipoxin inhibit inflammatory pain processing. J Exp Med 204: 245-252

\section{Etoricoxib causes fewer upper gastrointestinal adverse events than diclofenac}

Whether selective cyclo-oxygenase 2 inhibitors cause fewer gastrointestinal adverse events than traditional NSAIDs in patients who are also taking PPIs or cardioprotective aspirin remains unclear. Laine and colleagues, therefore, assessed the effects of the traditional NSAID diclofenac and the selective cyclo-oxygenase 2 inhibitor etoricoxib on gastrointestinal adverse events in participants from the Multinational Etoricoxib and Diclofenac Arthritis Long-term (MEDAL) program. 